\title{
Comparative Analysis of Anal Colposcopy with Histology in the Follow-Up of Patients under Treatment for Anal Condyloma
}

\author{
Thiago da Silveira Manzione ${ }^{1,2}$ Sidney Roberto Nadal2 ${ }^{20}$ Antônio José Gonçalves ${ }^{30}$ \\ ${ }^{1}$ Surgery Department, Irmandade da Santa Casa de Misericordia de \\ Address for correspondence Thiago da Silveira Manzione, MD, MSc, \\ São Paulo, São Paulo, SP, Brazil \\ 2 Instituto de Infectologia Emilio Ribas, São Paulo, SP, Brazil \\ ${ }^{3}$ Faculdade de Ciências Médicas, Santa Casa de São Paulo, São Paulo, \\ Av. Dr. Arnaldo, 165. CEP 01246-900, São Paulo/SP, Brazil \\ (e-mail: thiagomanzione@hotmail.com). \\ SP, Brazil \\ J Coloproctol 2022;42(1):63-67.
}

\begin{abstract}
Keywords

- anal colposcopy

- high-resolution anoscopy

- anal high-grade squamous intraepithelial lesion

- anal condyloma

Objectives To compare morphological abnormalities on anal colposcopy against histology to determine anal high-grade squamous intraepithelial lesions (HSILs).

Methods This is a retrospective data assessment of HIV-negative and HIV-positive patients undergoing outpatient follow-up. The sample comprised 54 patients presenting acetowhite lesions on anal colposcopy. Acetowhite lesions were classified according to their morphology into punctation, verrucous, mosaic, ulcerated, or hypervascularized, and biopsies of these specimens were classified as anal HSIL, low-grade squamous intraepithelial lesion (LSIL), or normal. The data were analyzed using SPSS for Windows version 13.0 (SPSS Inc., Chicago, IL, USA). The results were analyzed using the nonparametric Mann-Whitney test, the Fisher exact test and the chi-squared parametric test. A $95 \%$ confidence interval $(\mathrm{Cl})$ was used and a level of significance $<5 \%$ was adopted for all statistical tests.

Results Fifty-four patients ( 50 males, $80 \% \mathrm{HIV}+$ ) with biopsied acetowhite lesions were assessed. There were 31 punctation lesions, 1 classified as HSIL $(3.2 \%$; $95 \% \mathrm{Cl}$ : $0-$ 40.0), 17 verrucous lesions, $3 \mathrm{HSIL}$ (17.7\%; 95\%Cl: $0-10.7$ ), and 1 ulcerated, classified as HSIL (100\%), and 4 mosaic and 1 atypical vessel lesion, all classified as LSIL. The results showed no association of presence of anal HSIL with positivity for HIV infection or with counts above or below $500 / \mu \mathrm{l}$ in HIV+ patients. Statistical analysis was performed using the Mann-Whitney nonparametric test, the Fisher exact test, and the chi-squared parametric test.

Conclusion The comparison of morphological findings on anal colposcopy against histology revealed no morphological pattern suggesting anal HSIL.
\end{abstract}

received

July 16,2021

accepted after revision

August 23, 2021

published online

December 10, 2021
DOI https://doi.org/

10.1055/s-0041-1739299.

ISSN 2237-9363. (c) 2021. Sociedade Brasileira de Coloproctologia. All rights reserved.

This is an open access article published by Thieme under the terms of the Creative Commons Attribution-NonDerivative-NonCommercial-License, permitting copying and reproduction so long as the original work is given appropriate credit. Contents may not be used for commercial purposes, or adapted, remixed, transformed or built upon. (https://creativecommons.org/ licenses/by-nc-nd/4.0/)

Thieme Revinter Publicações Ltda., Rua do Matoso 170, Rio de Janeiro, RJ, CEP 20270-135, Brazil 


\section{Introduction}

The prevalence of anal squamous cell carcinoma (ASCC) has continued to rise over the decades. ${ }^{1}$

The high-risk group for ASCC includes patients with persistent HPV infection, immunosuppressive disorders, men who have sex with men (MSM), and women with a history of cervical or vaginal cancer. ${ }^{2-5}$

Anal low-grade squamous intraepithelial lesion (LSIL) is not considered a direct precursor of ASCC and can regress spontaneously or progress to anal high-grade squamous intraepithelial lesion (HSIL). ${ }^{6,7}$ In contrast, HSIL has less propensity to regress, particularly when containing highrisk oncogenic types, and is considered premalignant. ${ }^{7}$ Retrospective studies clearly show this evolution. ${ }^{8,9}$ Berry et al. $^{7}$ followed 138 HIV-positive men for 15 years and found that 27 patients developed ASCC at previous biopsy sites that had exhibited HSIL. In the high-risk group, this progression was estimated at 1 in 377 cases per year. $^{10}$

Anal colposcopy is the gold standard for identifying anal lesions for biopsy. ${ }^{11,12}$

Identifying these lesions on high-resolution anoscopy depends on the understanding and recognition of four main features: color tone and intensity of the acetowhite, margins and surface contour of the acetowhite areas, vascular features, and iodine staining pattern.

Anal LSILs are associated with less dense and less extensive acetowhite areas compared with HSILs, which are chalky-white, greyish-white in appearance and are thicker, dense, more extensive and have more complex lumen. ${ }^{13}$

When neoplasia develops as a result of HPV infection, the capillary system may be trapped into the dysplastic epithelium and a thin epithelium layer can cover these vessels. This forms the basis of punctate and mosaic blood vessel patterns. (-Figs. 1 A and $\mathbf{B}$ ).

Verrucous lesions ( - Fig. 1 C) have a characteristic aspect, exhibiting a papilla or arborescent, spiral, stacked vascular surface. These lesions can evolve to ulcers or atypical vessels that are associated with HSIL ( - Figs. $1 \mathbf{D}$ and $\mathbf{E}$ ).

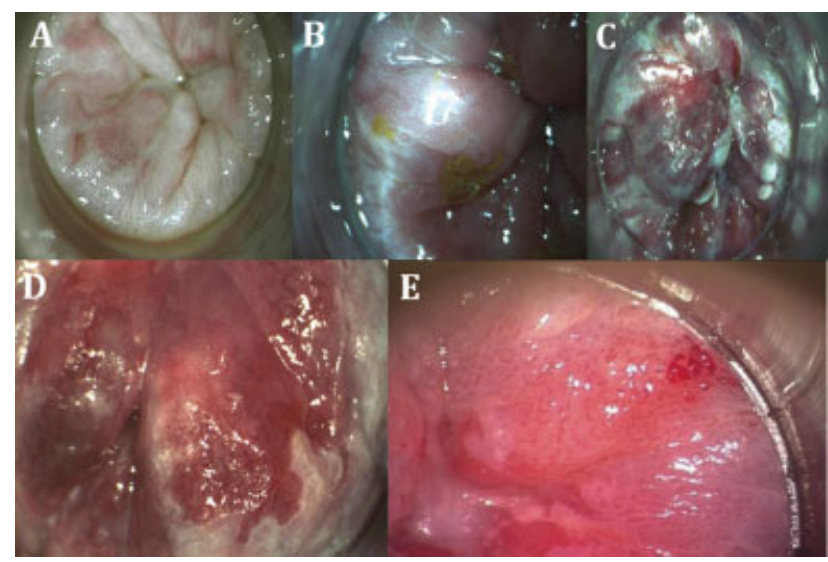

Fig. 1 Lesion: A. Punctation; B. Mosaic, C. Verrucous; D. Ulcerated; E. Atypical vessels.
Based on the premise that anal colposcopy is the gold standard for diagnosing subclinical lesions resulting from HPV, together with the steady rise in ASCC incidence, the high rates of neoplasia recurrence and the success of followup programs in reducing cervical cancer, the present study compared changes on anal colposcopy against histology findings, given that these changes are used to guide the selection of lesions for biopsy, thereby helping to reduce the incidence of ASCC.

The objective of the present study was to compare morphological changes on anal colposcopy against histology to diagnose anal high-grade squamous intraepithelial neoplasia in patients without clinical lesions.

\section{Casuistic}

\section{Casuistic}

A retrospective assessment data of HIV-negative and HIVpositive patients undergoing outpatient follow-up after eradication of clinical HPV-associated anal lesions was performed. Patients treated between January 2014 and July 2017 were included.

\section{Sample Characteristics}

The sample comprised 54 patients presenting acetowhite lesions on follow-up anal colposcopy 30 days after management of condyloma acuminata of the anal margin and/or canal.

\section{Inclusion Criteria}

The sample included patients $>18$ years old exhibiting biopsied acetowhite lesions on follow-up anal coloscopy.

\section{Methods}

The present study was approved by the Research Ethics Committee of the Instituto de Infectologia Emílio Ribas (IIER) under the opinion number 2,845,945, and written informed consent was obtained from all subjects.

\section{Anal Coloscopy}

A conventional colposcope (Medpej 7000, binocular with 7$25 \mathrm{X}$ optic lenses) was employed for the tests. The test method entailed the following standard procedure: application of $3 \%$ acetic acid to the anal margin and canal; examination of the region after 2 minutes at different magnifications ( $5 \mathrm{x}$ to $40 \mathrm{x}$ ) for acetowhite areas. Acetowhite lesions were biopsied, and the specimens were sent to the Pathologic Anatomy Laboratory. The specimens were classified as anal HSIL, LSIL or normal.

\section{Findings on Anal Coloscopy}

Findings were classified as negative, in the absence of changes, or as positive, in the presence of acetowhite areas and/or of areas not stained with iodine solution. These lesions were then classified according to their morphology into punctation, verrucous, mosaic, ulcerated or hypervascularized. 
Table 1 Distribution of 54 patients submitted to anal coloscopy, by gender and HIV-positivity

\begin{tabular}{|l|l|l|l|}
\hline HIV infection & Negative & Positive & Total \\
\hline Female & $0(0.0 \%)$ & $4(100 \%)$ & $4(100 \%)$ \\
\hline Male & $10(20 \%)$ & $40(80 \%)$ & $50(100 \%)$ \\
\hline Total & $10(18.5 \%)$ & $44(81.5 \%)$ & $54(100 \%)$ \\
\hline
\end{tabular}

Instituto de Infectologia Emílio Ribas, São Paulo, 2020.

\section{Statistical Analysis}

The data were analyzed using SPSS for Windows version 13.0 (SPSS Inc., Chicago, IL, USA). The results were analyzed using the nonparametric Mann-Whitney test, the Fisher exact test, and the chi-squared parametric test. A 95\% confidence interval $(\mathrm{CI})$ was used and a level of significance $<5 \%$ was adopted for all statistical tests.

\section{Results}

\section{Gender}

The results of the statistical analysis using the Fisher exact test revealed that both the HIV- positive and -negative sample was predominantly male. (-Table $\mathbf{1}$ ).

\section{Age}

Statistical analysis using the Mann-Whitney test showed that HIV-positive patients were older than HIV-negative patients $(p=0.005)$.

\section{Morphological Findings of Acetowhite Lesions Disclosed by Anal Colposcopy}

Histological Results of Acetowhite Lesions Disclosed by Anal Colposcopy

Characteristics of the groups: (-Table 2).

\section{Punctation Acetowhite Lesion}

This lesion group contained 31 (57.5\%) patients, comprising 1 woman and 30 men. The age of the patients ranged from 18

Table 2 Distribution of results according to morphological features of acetowhite lesions disclosed on anal colposcopy and respective histology results

\begin{tabular}{|l|l|l|l|l|}
\hline \multirow{2}{*}{$\begin{array}{l}\text { Morphology of } \\
\text { acetowhite lesions }\end{array}$} & \multicolumn{4}{l|}{ Histology } \\
\cline { 2 - 5 } & Normal & Anal LSIL & Anal HSIL & Total \\
\hline Punctation & $8(25.8 \%)$ & $22(71 \%)$ & $1(3.2 \%)$ & $31(100 \%)$ \\
\hline Verrucous & $4(23.5 \%)$ & $10(58.8 \%)$ & $3(17.7 \%)$ & $17(100 \%)$ \\
\hline Mosaic & $2(50 \%)$ & $2(50 \%)$ & 0 & $4(100 \%)$ \\
\hline Ulcerated & 0 & 0 & $1(100 \%)$ & $1(100 \%)$ \\
\hline Atypical Vessels & $1(100 \%)$ & 0 & 0 & $1(100 \%)$ \\
\hline
\end{tabular}

Abbreviations: LSIL, low-grade squamous intraepithelial lesion; HSIL, high-grade squamous intraepithelial lesion.

Chi-squared $=0.167$.

Instituto de Infectologia Emílio Ribas, São Paulo, 2020.

No statistical significance was detected, i.e., no morphological type had higher prevalence of high grade.
Table 3 Acetowhite lesions disclosed on anal colposcopy according to morphological features and respective histology results for HSIL (\%) and 95\% confidence interval

\begin{tabular}{|l|l|l|l|l|}
\hline $\begin{array}{l}\text { Morphology of } \\
\text { acetowhite lesions }\end{array}$ & \multirow{2}{*}{$n$} & \multirow{2}{*}{$\%$} & \multicolumn{2}{|l|}{$95 \% \mathrm{Cl}$} \\
\cline { 4 - 5 } & & & LB & UB \\
\hline Verrucous & 17 & 17.6 & 0.0 & 40.4 \\
\hline Punctation & 31 & 3.2 & 0.0 & 10.7 \\
\hline
\end{tabular}

Abbreviations: $\mathrm{Cl}$, confidence interval; LB, lower bound; UB, upper bound.

Verrucous lesion: proportionally greater rate of HSIL (17.6\%), ranging from zero to $40 \%$; - Punctation lesion: rate of HSIL $3.2 \%$, ranging from 0 to $10.7 \%$; Fisher's exact test $(p=1.0)$, non-significant.

Instituto de Infectologia Emílio Ribas, São Paulo, 2020.

to 63 years old, with a mean of 40.5 years old. Six patients were HIV-negative and 25 were HIV-positive.

\section{Verrucous Acetowhite Lesion}

This lesion group contained 17 (31.5\%) patients, comprising 2 women and 15 men. The age of the patients ranged from 23 to 63 years old, with a mean of 43 years old. Four patients were HIV-negative and 13 were HIV-positive.

\section{Mosaic Acetowhite Lesion}

This lesion group contained 4 (7.4\%) patients, comprising 1 woman and 3 men. The age of the patients ranged from 30 to 56 years old, with a mean of 43 years old. All patients in this group were HIV-positive.

\section{Ulcerated Acetowhite Lesion}

This group consisted of 1 (1.8\%) 51-year-old male patient who was HIV-positive.

\section{Atypical Vessel Acetowhite Lesion}

This group consisted of 1 (1.8\%) 50-year-old male patient who was HIV-positive (-Table 3 ).

Table 4 Distribution of 44 HIV-positive patients submitted to anal coloscopy with biopsy of acetowhite lesions, according to CD4 lymphocyte count above and below $500 / \mu \mathrm{L}$ and presence of HSIL

\begin{tabular}{|l|l|l|l|}
\hline & \multicolumn{3}{|l|}{ Histology } \\
\hline & Non-high grade & High-grade & Total \\
\hline CD4 T cell count & (Normal + LSIL) & HSIL & \\
\hline$\leq 500 / \mu \mathrm{l}$ & 19 & 2 & 21 \\
\hline$>500 / \mu \mathrm{l}$ & 21 & 2 & 23 \\
\hline Total & 40 & 4 & 44 \\
\hline
\end{tabular}

Abbreviations: HSIL, high-grade squamous intraepithelial lesion; LSIL, lowgrade squamous intraepithelial lesion.

Fisher exact test $(p=1.0)$, nonsignificant, thus confirming CD4 lymphocyte T cell count was not a determinant factor in the development of HSIL.

Instituto de Infectologia Emílio Ribas, São Paulo, 2020. 


\section{CD4 Lymphocyte Count}

Results of the Fisher exact test $(=1)$ revealed no association between the presence of HSIL and counts above or below $500 / \mu$ in HIV + patients (-Table 4).

\section{Discussion}

The present study sample of HIV-positive individuals contained more men than women. The study results can be explained by the fact that HPV-associated anal lesions can be more common in men who have sex with men (MSM) and who are also HIV-positive, as outlined previously.

The HIV-positive group represented $81.5 \%$ of the population assessed. The high rate of HIV-positive patients may be due to the fact that the IIER is a referral service for AIDS.

Regarding age, the mean age of the individuals was 39.4 years old, with a wide range between minimum and maximum, findings which are consistent with the literature, showing high rates of HPV-associated anal lesions across all ages, from 18 to 50 years old. ${ }^{14}$

The HIV-positive patients were older than their HIVnegative counterparts. Studies have shown that immunosuppression due to HIV tends to perpetuate infection by HPV, increasing the risk of lesions caused by the virus with age. ${ }^{15,16}$

Anal colposcopy is a simple, noninvasive exam that can be used in high-risk patients for diagnosing ASCC. A recent study by Palefsky et al. ${ }^{17}$ reported a large series of patients involving 571 cases. A total of 835 lesions were described, biopsied and correlated with histology. Four out of the 5 categories (contour, surface, vascular patterns, and Lugol staining) clearly distinguished HSIL from LSIL. ${ }^{18}$ A study by Jay et al. ${ }^{19}$ involving 152 cases and 385 biopsies also found similarities in the colposcopic appearance of anal lesions compared with cervical lesions and in correlation with their expected dysplastic grade.

In the present study, acetowhite lesions were classified according to their predominant morphology into punctation, verrucous, mosaic, ulcerated or atypical vessels.

The examinations revealed 31 (57.5\%) punctation, 17 (31.5\%) verrucous, 4 (7.4\%) mosaic, 1 (1.8\%) ulcerated, and $1(1.8 \%)$ atypical vessel lesion. The high incidence of verrucous lesions (31.5\%) might be attributed to the fact that, unlike most studies in which anal colposcopy of the anal canal alone is performed, the present study also assessed the anal margin, the perineum and the coccyx region, sites where verrucous lesions are more prevalent. ${ }^{20}$ In addition, the predominant morphology of the lesion was also taken into account when this was mixed (e.g., verrucous + punctation and/or mosaic) and verrucous lesions are more visible and overlay flat lesions (punctation and mosaic).

On histology, lesions were normal in 15 (27.7\%) cases, LSIL in 34 (63.1\%), and HSIL in 5 (9.2\%). This result was congruent with the study objective of biopsying the areas with abnormal colposcopic appearance, irrespective of whether lesions were suggestive of HSIL or otherwise.

Detection of a higher rate of HSIL in punctation (3.1\%) and mosaic (no cases) lesions was expected. However, the results revealed that verrucous lesions, classically correlated with LSIL, exhibited the highest rate of HSIL (17.7\%). These results are noteworthy in that they contradict the literature, particularly regarding evidence related to the cervix. In the present analysis, punctation and mosaic lesions were not classified into fine punctation/mosaic, normally associated with lowgrade lesions, or into punctation/coarse mosaic, typically associated with high-grade lesions. Possibly, most of the punctation lesions and all mosaic lesions biopsied were fine changes.

This subclassification was not done because the anatomical characteristics of the anal canal render detailed visualization of lesions difficult. This does not rule out the possibility of more precise classification of subclinical lesions during anal coloscopy but highlights the need for the appropriate equipment and a high skill level of the colposcopist. $^{18}$

The current findings corroborate those in the literature showing that $\sim$ between 10 and $14 \%$ of lesions with LSIL appearance on anal colposcopy have HSIL histology, ${ }^{18}$ where this differentiation can be aided by applying Lugol staining.

High-grade squamous intraepithelial lesions are more common among individuals in high-risk groups. In the present study, HIV-positive patients had higher HSIL rates, although this difference did not reach statistical significance. Likewise, no statistical difference was found for CD4 lymphocytes $\mathrm{T}$ cell count above or below $500 / \mu \mathrm{l}$, confirming that immunity was not a determinant.

Experience administering colposcopy is an important factor, but even highly skilled colposcopists can take biopsy samples of lesions suggestive of HSIL that are subsequently not confirmed histologically. This indicates that a deeper understanding of the features of subclinical lesions on anal colposcopy could improve the likelihood of biopsying highergrade lesions.

Ideally, lesions should be treated while still in their early stages and before progressing to high-grade clinical lesions. The detection of a higher rate of normal and LSIL lesions associated with the absence of ASCC in the present cohort shows this objective is being achieved.

\section{Conclusion}

The present study found no specific morphological pattern suggestive of HSIL.

Financial Support

None.

Conflict of Interests

The authors have no conflict of interests to declare.

\section{References}

1 Siegel RL, Miller KD, Jemal A. Cancer statistics, 2019. CA Cancer J Clin 2019;69(01):7-34

2 Daling JR, Madeleine MM, Johnson LG, et al. Human papillomavirus, smoking, and sexual practices in the etiology of anal cancer Cancer 2004;101(02):270-280 
3 Jiménez W, Paszat L, Kupets R, Wilton A, Tinmouth J. Presumed previous human papillomavirus (HPV) related gynecological cancer in women diagnosed with anal cancer in the province of Ontario. Gynecol Oncol 2009;114(03):395-398

4 Sunesen KG, Nørgaard M, Thorlacius-Ussing O, Laurberg S. Immunosuppressive disorders and risk of anal squamous cell carcinoma: a nationwide cohort study in Denmark, 1978-2005. Int J Cancer 2010;127(03):675-684

5 Frisch M, Biggar RJ, Goedert JJ. Human papillomavirus-associated cancers in patients with human immunodeficiency virus infection and acquired immunodeficiency syndrome. J Natl Cancer Inst 2000;92(18):1500-1510

6 Palefsky JM. Anal human papillomavirus infection and anal cancer in HIV-positive individuals: an emerging problem. AIDS 1994;8 (03):283-295

7 Berry JM, Jay N, Cranston RD, et al. Progression of anal high-grade squamous intraepithelial lesions to invasive anal cancer among HIV-infected men who have sex with men. Int J Cancer 2014;134 (05):1147-1155

8 Scholefield JH, Castle MT, Watson NF. Malignant transformation of high-grade anal intraepithelial neoplasia. Br J Surg 2005;92 (09):1133-1136

9 Kreuter A, Potthoff A, Brockmeyer NH, et al; German Competence Network HIV/AIDS. Anal carcinoma in human immunodeficiency virus-positive men: results of a prospective study from Germany. Br J Dermatol 2010;162(06):1269-1277

10 Machalek DA, Poynten M, Jin F, et al. Anal human papillomavirus infection and associated neoplastic lesions in men who have sex with men: a systematic review and meta-analysis. Lancet Oncol 2012;13(05):487-500

11 Instituto Nacional de Câncer (Brasil)- Ministério da Saúde. Diretrizes brasileiras para o rastreamento do câncer do colo do útero. Coordenação Geral de Ações Estratégicas. Divisão de Apoio à Rede de Atenção Oncológica. - Rio de Janeiro: INCA, 2011. Accessed July 21, 2020 at: https://www.inca.gov.br/sites/ufu.sti.inca.local/ files//media/document//diretrizes-para-rastreamento_cancer_ colo_utero-2011.pdf

12 Nadal SR, Manzione CR. Uso do colposcópio para avaliar a região perianal e o canal anal - padronização técnica da nomenclatura e indicações. Rev Bras Coloproctol 2004;24:379-381

13 Sellors JW, R. Sankaranarayanan R. Avaliação colposcópica da neoplasia intraepitelial cervical. Colposcopia e tratamento da neoplasia intra-epitelial cervical: Manual para principiantes, Capítulo 7, 2004:55-68. Accessed July 21, 2020 at: https:// screening.iarc.fr/doc/colpoptmanual.pdf

14 Chin-Hong PV, Vittinghoff E, Cranston RD, et al. Age-Specific prevalence of anal human papillomavirus infection in HIV-negative sexually active men who have sex with men: the EXPLORE study. J Infect Dis 2004;190(12):2070-2076

15 de Pokomandy A, Rouleau D, Ghattas G, et al. HAART and progression to high-grade anal intraepithelial neoplasia in men who have sex with men and are infected with HIV. Clin Infect Dis 2011;52(09):1174-1181

16 Dietz CA, Nyberg CR. Genital, oral, and anal human papillomavirus infection in men who have sex with men. J Am Osteopath Assoc 2011;111(3, Suppl 2)S19-S25

17 Palefsky JM, Holly EA, Hogeboom CJ, Berry JM, Jay N, Darragh TM. Anal cytology as a screening tool for anal squamous intraepithelial lesions. J Acquir Immune Defic Syndr Hum Retrovirol 1997;14 (05):415-422

18 Jay N, Berry JM, Miaskowski C, et al. Colposcopic characteristics and Lugol's staining differentiate anal high-grade and low-grade squamous intraepithelial lesions during high resolution anoscopy. Papillomavirus Res 2015;1:101-108

19 Jay N, Berry JM, Hogeboom CJ, Holly EA, Darragh TM, Palefsky JM. Colposcopic appearance of anal squamous intraepithelial lesions: relationship to histopathology. Dis Colon Rectum 1997;40(08): 919-928

20 Koutsky L. Epidemiology of genital human papillomavirus infection. Am J Med 1997;102(5A):3-8 